Paidéia, 2006, 16(35), 337-348

\title{
INTELIGÊNCIA EMOCIONAL E DESEMPENHO NO TRABALHO: UM ESTUDO COM MSCEIT, BPR-5 E 16PF ${ }^{1}$
}

\author{
Cláudia Cobêro \\ Ricardo Primi ${ }^{2}$ \\ Monalisa Muniz \\ Universidade São Francisco
}

\begin{abstract}
Resumo: O presente trabalho teve como objetivo investigar a validade de uma medida de inteligência emocional correlacionando-a com medidas de inteligência, personalidade e desempenho profissional. Participaram do estudo 119 sujeitos, com idade entre 17 e 64 anos, de ambos os sexos e que trabalham em empresas situadas em municípios do interior do estado de São Paulo. Os instrumentos utilizados foram: Versão em Português do Mayer-Salovey-Caruso-Emotional Intelligence Test (MSCEIT), o Questionário Dezesseis Fatores da Personalidade (16PF), Bateria de Provas de Raciocínio (BPR-5), Avaliação de Desempenho respondido por duas pessoas (um supervisor e um colega). Os resultados apontam baixa correlação entre inteligência emocional e personalidade, bem como com inteligência. Indicam também que a faceta regulação das emoções se correlaciona com o desempenho profissional e apresenta validade incremental em relação à inteligência. Em suma conclui-se que a inteligência emocional constitui um tipo diferenciado de inteligência útil na avaliação psicológica no contexto organizacional.
\end{abstract}

Palavras-chave: Inteligência Emocional; Mayer-Salovey-Caruso-Emotional Intelligence Test (MSCEIT); Avaliação Psicológica; Processos Seletivos; BPR-5; 16PF; Validade.

\section{EMOTIONAL INTELLIGENCE AND JOB PERFORMANCE: A STUDY WITH MSCEIT, BPR-5 AND 16PF}

Abstract: This research had as objective to investigate the validity of a emotional intelligence measure correlating it with measures of intelligence, personality, and job performance. The participants were 119 subjects, aged 17 to 64 years old, of bothe sex that work in cities industries from the state of São Paulo. The instruments used: the Mayer-Salovey-Caruso-Emotional Intelligence Test (MSCEIT), protuguese version, the Sixteen Personality Factors Questionnaire (16PF), the Battery of Reasoning (BPR-5) a performance evaluation made by two persons (supervisor and a colegue). The results show low correlations between emotional intelligence and personality. Also that the branch managing emotions is correlated with job performance and presents validy with the intelligence. In sum it was concluded that emotional intelligence constitutes a differentiated type of intelligence useful for psychological assessment in the context of industrial and organizational psychology.

Key words: Emotional Intelligence; Mayer-Salovey-Caruso-Emotional Intelligence Test (MSCEIT); Psychological Assessment; Selection Processes; BPR-5; 16PF; Validity.

O termo Inteligência Emocional tornou-se conhecido na década de 90 pela obra de Daniel Goleman (1995) intitulada "Inteligência Emocional".

\footnotetext{
${ }^{1}$ Recebido em 04/12/2006 e aceito para publicação em 06/02/2007.

2 Endereço para correspondência: Ricardo Primi, Universidade São Francisco, Laboratório de Avaliação Psicológica e Educacional (LabAPE), Rua Alexandre Rodrigues Barbosa, 45, CEP: 13251-900, Itatiba-SP, E-mail: ricardo.primi@saofrancisco.edu.br ou rprimi@uol.com.br
}

Logo após o lançamento desse livro, o termo foi rapidamente disseminado em diversos segmentos da sociedade (Goleman, 1995; Roberts, Flores-Mendoza \& Nascimento, 2002). Mas, ao contrário do pensado, esse conceito não foi proposto por Daniel Goleman, mas por Peter Salovey e John Mayer em 1990.

Segundo Mayer, Salovey e Caruso (2000), a relação teórica entre inteligência e emoção já era 


\section{Cláudia Cobêro}

usada antes da divulgação do termo inteligência emocional no início da década de 90, sendo utilizado pela primeira vez por Payne (1985, citado por Hein, 2003) em sua tese de doutorado, em que ele apresenta uma estrutura teórica fundamentada e discute a emoção e a inteligência emocional do ponto de vista filosófico, sem dar ênfase à demonstração empírica de suas idéias, o que levou a pouca receptividade do modelo.

Do ponto de vista teórico empírico, o termo inteligência emocional foi utilizado pela primeira vez por, Mayer, DiPaolo e Salovey (1990), em um periódico científico internacional de Psicologia, num trabalho que teve como objetivo estudar empiricamente um de seus componentes, a habilidade de percepção de conteúdos afetivos. Essa pesquisa citou a inteligência emocional como uma subclasse da Inteligência Social, cujas habilidades estariam relacionadas ao "monitoramento dos sentimentos em si e nos outros, na discriminação entre ambos e na utilização desta informação para guiar o pensamento e as ações", segundo Salovey e Mayer (1990), que ao publicarem um segundo artigo, este se tornou mais conhecido que o primeiro, por apresentar o conceito inteligência emocional.

Nele, os autores fizeram uma análise de pesquisas científicas que justificavam a necessidade de se conceber a existência de uma habilidade relativamente distinta, que referiram como inteligência emocional e procuraram posicioná-la como um subconjunto da inteligência social e das inteligências múltiplas de Gardner (Salovey \& Mayer, 1990);0 modelo apresentado envolvia as habilidades de: a) avaliação e expressão de emoções em si mesmo e no outro de maneira verbal e não verbal; b) regulação de emoções em si mesmo e no outro por meio da empatia e c) utilização de emoções por meio de um planejamento flexível do pensamento criativo, do redirecionamento da atenção e da motivação.

Ao longo da década de noventa o conceito passou por revisões sendo que a definição atual diz que a inteligência emocional refere-se "a capacidade de perceber acuradamente, de avaliar e de expressar emoções; a capacidade de perceber e/ou gerar sentimentos quando eles facilitam o pensamento; a capacidade de compreender a emoção e o conhe- cimento emocional; e a capacidade de controlar emoções para promover o crescimento emocional e intelectual" (Mayer \& Salovey, 1997).

Após as primeiras publicações sobre o tema (Mayer, DiPaolo \& Salovey, 1990; Salovey \& Mayer, 1990), muitas críticas foram feitas, apontando principalmente que se tratava de uma metáfora inapropriada e enganosa, na qual os autores estariam reescrevendo a inteligência social e que não existia nenhuma habilidade importante relacionada à emoção. Em um editorial da revista Intelligence, Mayer e Salovey (1993) respondem a essas críticas definindo a inteligência emocional como a capacidade de processar informações carregadas de afeto, diferenciando-a assim das definições da social, apresentam também seus mecanismos e propõem o termo inteligência emocional para estudos que investigam a interação entre emoção e inteligência.

Segundo Goleman (1995) "o QI e a inteligência emocional não são capacidades que se sobrepõem, mas distintas. Na verdade, há uma ligeira correlação entre QI e alguns aspectos da inteligência emocional, embora bastante pequena para que fique claro que se trata de duas entidades bastante independentes" (p. 57). Mayer e Salovey (1997) dizem também que a inteligência emocional não é o oposto da inteligência, mas sim a intersecção entre ela e a emoção. Assim, ela seria uma habilidade cognitiva relacionada ao uso das emoções para ajudar na resolução de problemas; argumentam ser inadequado conceber a emoção sem inteligência, ou esta sem aquela, trazendo o conceito uma visão integrada da razão e emoção.

Mayer e Salovey (1997) definem esse construto por quatro ramificações dos processos psicológicos, organizadas em níveis de complexidade, dos mais elementares aos mais complexos e psicologicamente mais integrados:

-A primeira diz respeito à percepção, avaliação e expressão da emoção, referindo-se a capacidade de identificar emoções em si e em outras pessoas, desenhos, objetos e paisagem mediante linguagem, sons, aparência e comportamento, abrangendo também a capacidade de expressar emoções e necessidades relacionadas com sentimentos, bem como de discernir entre expressões falsas e verdadeiras. 
-A segunda refere-se à emoção como facilitadora do ato de pensar, a possível facilitação do pensamento quando, por exemplo, as emoções priorizam certas idéias dirigindo a atenção para informações mais importantes; e também gerandoas de maneira relativamente voluntária para poder examinar as informações contidas nessas experiências emocionais de tal forma a ajudar o julgamento de situações que as envolvem.

-A terceira, chamada compreensão e análise de emoções; emprego do conhecimento emocional refere-se à capacidade de rotular emoções, de interpretar os significados que elas trazem sobre os relacionamentos interpessoais, de compreender as complexas e de reconhecer transições mais comuns entre elas.

-A quarta e última ramificação é o controle reflexivo de emoções para promover o crescimento emocional e intelectual, referindo-se à capacidade de se manter aberto a sentimentos, agradáveis ou desagradáveis, adminsitrando a emoção em si mesmo e nos outros pela moderação das negativas e valorização das agradáveis, sem que haja repressão ou exagero dos estados psicológicos que elas podem provocar (Mayer \& Salovey, 1997).

Um dos questionamentos centrais da literatura sobre inteligência emocional é se realmente este tipo de inteligência difere das já pesquisadas, ou se ela não estaria ligada aos traços de personalidade. Diante disso, a pergunta é "será que as capacidades referidas no conceito inteligência emocional se constituem realmente em algo novo que não podem ser explicadas por construtos já conhecidos como as inteligências tradicionais e a personalidade?"

Embora existam pesquisadores trabalhando no conceito de inteligência emocional, os estudos que buscam evidências empíricas de que ela realmente existe e que difere dos traços de personalidade e da inteligência proposta há décadas são escassos, principalmente no Brasil, em que duas pesquisas foram realizadas buscando investigar as correlações entre inteligência emocional e a tradicional e mostraram que elas são baixas, o que é de fato esperado entre esses construtos (Bueno, 2002; Jesus Junior, 2004). Já com relação à personalidade estudos realizados indicam maior divergência do que convergência entre as medidas de inteligência emocional e traços de personalidade (Bueno, 2002; Bueno \& Primi, 2003; Dantas, 2004, Nascimento, 2006 ; Primi, Bueno \& Muniz, 2006).

Os resultados encontrados nos estudos brasileiros corroboram os das pesquisas estrangeiras, como os relatados por Mayer, Caruso e Salovey (2000); o mesmo com relação às correlações com personalidade relatados em Bedwell (sd.), Brackett e Mayer (2003), Lopes, Bracket, Nezlek, Schutz e Salovey (2003), Mayer, Salovey, Caruso e Sitarenios (2001, 2003).

Paralelamente a esses estudos o conceito se disseminou rapidamente no contexto organizacional tornando-se popular como característica desejável e preditora de sucesso generalizado. Como frequentemente ocorre, essa disseminação aconteceu independente das informações empíricas que sustentassem a utilidade desse construto para a previsão do desempenho, numa popularização em razão das idéias um tanto exageradas apresentadas por Goleman (1995) dizendo que a inteligência emocional seria a capacidade mais importante na explicação do sucesso no trabalho, levando empresas a investir em treinamentos e alterações de suas práticas seletivas e de mudanças de cargos baseandose na idéia de que pessoas com alta inteligência emocional apresentariam um desempenho mais eficaz em seu trabalho, assumindo a existência do construto e da sua utilidade, sem, entretanto, se basear, para isto em dados empíricos. Mais preocupante é o fato de inventários e escalas de inteligência emocional serem usados na seleção de pessoal sem que se tenha certeza sobre sua eficácia.

Segundo Goleman (1995) o desenvolvimento do QE (coeficiente emocional), além do QI, é cada vez mais importante para o sucesso e sobrevivência das empresas. Para Roberts, Flores-Mendoza e Nascimento (2002) esse amplo interesse das empresas pela inteligência emocional pode estar vinculado a uma conjectura de que pessoas com melhor gerenciamento de suas próprias emoções são possivelmente as melhores sucedidas no mercado de trabalho e que acabam também por ter mais qualidade de vida. Seria então a Inteligência Emocional um preditor de desempenho profissional? Mas que evidências empíricas baseiam essa afirmação? 


\section{Cláudia Cobêro}

O que de fato os estudos empíricos demonstram é que o fator geral de inteligência consiste no preditor único mais importante do desempenho no trabalho (Kuncel, Hezlett \& Ones, 2004; Wilhelm \& Engle, 2005), ou seja, nenhuma outra variável sozinha consegue atingir o seu nível de predição. Para Barrett e Depinet (1991) o g é um preditor eficaz do desempenho em muitas ocasiões ocupacionais, visto que sua relação tem permanecido estável ao longo do tempo; para os mesmos autores, a relação positiva entre a inteligência e o sucesso no trabalho não estabelece um artifício decorrente do nível socioeconômico, mas sim, de que a inteligência é a causa fundamental da correlação entre nível socioeconômico e sucesso ocupacional. Análises em pesquisas de Brody (1997, conforme citado por Nascimento, 2000) com relação ao nível ocupacional e habilidades intelectuais demonstraram que variações na inteligência influenciam mudanças no status ocupacional.

A literatura é abundante em demonstrar as correlações entre inteligência tradicionalmente definida e a mensurada com o desempenho no trabalho. E a emocional teria ou não essa correlação? Em caso afirmativo ela seria explicada por um fator específico de inteligência (ligado ao processamento das emoções) ou por cognitivos já conhecidos? E ela teria maior magnitude do que a observada entre o fator $\mathrm{g}$ e desempenho?

Assim o objetivo deste estudo foi verificar se inteligência emocional é capaz de predizer o desempenho profissional para além do que a tradicional possa prever, ou seja, esse estudo buscou trazer dados de validade incremental (Smith, Fischer $\&$ Fister, 2003). Também averiguar a relação entre inteligência emocional e personalidade, já que estas são questões discutidas no meio científico que muitas vezes critica esse construto como apenas um novo rótulo para traços de personalidade.

De maneira geral a carência de instrumentos validados no contexto organizacional no Brasil justifica parte desse trabalho, que busca a validade de critério para a inteligência emocional correlacionando-a com

\footnotetext{
${ }^{3}$ Participaram desse estudo os que assinaram o termo de consentimento.
}

um instrumento de medida de desempenho profissional. Os procedimentos de validação de critério indicam a efetividade de um teste para predizer o desempenho de um indivíduo em atividades específicas, fornecendo informações pela validade de critério, que são relevantes para os testes usados na seleção e classificação de pessoal (Anastasi \& Urbina, 2000).

\section{Método}

\section{Participantes}

Participaram deste estudo 119 profissionais atuantes em empresas de diversos segmentos, de pequeno, médio e grande porte, situadas em municípios do interior do estado de São Paulo. Da amostra 65,8\% eram do sexo masculino e $34,2 \%$ do feminino, com idade variando entre 17 e 64 anos, numa média de 30 anos; sua escolha foi feita por conveniência e agrupados segundo sua área de atuação profissional, a saber: $17,60 \%$ da administrativa, $14,30 \%$ da comercial e $68,10 \%$ da produção industrial. Os participantes apresentavam tempo de empresa variando entre 1 a 228 meses, com uma média igual a 44,27 meses $^{3}$.

\section{Instrumentos}

O instrumento (Mayer, Salovey, Caruso Emotional Intelligence Test - MSCEIT Mayer, Salovey, Caruso, 2002b) ficou composto por 141 itens, distribuídos em 8 seções conforme mostra o Quadro 1. As seções A (faces) e E (figuras) são destinadas a avaliação da capacidade de perceber emoções em faces e paisagens, respectivamente; as B (facilitação) e F (sensação) são compostas por tarefas ligadas à utilização da emoção para facilitação do pensamento; a compreensão de emoções é avaliada pelas tarefas propostas nas C (transição) e $\mathrm{G}$ (mistura); finalmente, o gerenciamento das emoções é avaliado por meio das tarefas da D (administração de emoções) e H (Relações emocionais). O Quadro 1 traz os diferentes níveis de combinação dos subtestes para dar escores nas quatro facetas, duas áreas e um geral, utilizando-se nesse estudo escores dos subtestes em algumas análises e das áreas em outras. 
Quadro 1. Organização dos sub-testes do MSCEIT

\begin{tabular}{|c|c|c|c|c|}
\hline Seção & Sub-Teste & Faceta & Área & Escala Geral \\
\hline A & Faces & Percepção das & \multirow{4}{*}{$\begin{array}{c}\text { Experiencial } \\
\text { (IEE) }\end{array}$} & \multirow{8}{*}{$\begin{array}{l}\text { Inteligência } \\
\text { Emocional }\end{array}$} \\
\hline E & Figuras & emoções & & \\
\hline $\mathrm{B}$ & Facilitação & Facilitação do & & \\
\hline $\mathrm{F}$ & Sensações & pensamento & & \\
\hline $\mathrm{C}$ & Transições & Compreensão & \multirow{4}{*}{$\begin{array}{c}\text { Estratégica } \\
\text { (IES) }\end{array}$} & \\
\hline $\mathrm{G}$ & Misturas & das emoções & & \\
\hline $\mathrm{D}$ & $\begin{array}{l}\text { Administração } \\
\text { de Emoções }\end{array}$ & \multirow{2}{*}{$\begin{array}{l}\text { Gerenciamento } \\
\text { das emoções }\end{array}$} & & \\
\hline $\mathrm{H}$ & $\begin{array}{l}\text { Relações } \\
\text { Emocionais }\end{array}$ & & & \\
\hline
\end{tabular}

O subteste Faces é composto por quatro grupos de itens; os participantes vêem várias faces e para cada uma respondem numa escala de cinco pontos, indicando o grau que determinada emoção está presente nela. O Figuras é formado por seis grupos, em que o indivíduo indica, numa escala de cinco pontos discriminada com faces de emoção, o quanto cada uma delas está presente em certa paisagem ou desenho abstrato. O Sensação consta de cinco grupos de tarefas com três respostas cada, em que estas criam uma emoção e associam uma sensação a ela, por exemplo, uma situação com sentimento de culpa, e a pessoa tem de decidir o quanto isso se parece com as sensações "doce ou amargo". O Facilitação apresenta cinco grupos de itens com três respostas cada, e o participante julga qual seria a emoção que poderia ajudar a pensar em soluções para certa situação, como o quanto a emoção raiva ajudaria na composição de uma marcha militar. O Misturas tem 12 itens, que solicitam do participante quais emoções podem ser combinadas para formar outra emoção, como qual resultaria da combinação de aceitação e preocupação. O Transição é composto por 20 itens, nos quais os participantes escolhem a emoção na seqüência apresentada, como, a depressão em razão do aumento da intensidade de tristeza. O Administração de emoções é formado por cinco grupos de itens com quatro respostas cada, em que os participantes julgam as ações mais efetivas para obter ou manter um estado emocional em determinada situação. Por fim, o Relações emocionais é constituído por três grupos de itens com três respostas cada, em que a pessoa julga qual ação seria mais efetiva para controlar os sentimentos de outra pessoa.

Esse teste foi corrigido pelo critério de consenso, identificando o item mais escolhido pela amostra, bem como sua freqüência, sendo que cada item passa a receber a pontuação igual à frequiência. Por exemplo, se no A a pontuação 1 obteve um percentual de $40 \%$, ele leva a pontuação igual a 40 , valendo-se da regra para os itens que compõem o teste MSCEIT.

A BPR 5 (Bateria de Provas de Raciocínio, (Primi \& Almeida, 1998) instrumento para a avaliação simultânea do raciocínio geral e das aptidões, para fornecer "informações sobre o funcionamento cognitivo das pessoas" (Primi \& Almeida, 2000a) em cinco áreas: raciocínio abstrato, verbal, visual-espacial, numérico e mecânico, apresentada em duas formas: A e B, sendo a A destinada a participantes de $6^{\mathrm{a}}$ a $8^{\mathrm{a}}$ séries de escolaridade, e a "B" aos de escolaridade superior aos da "A". As duas formas (A e B) são compostas por cinco subtestes, cada um organizado em caderno individual a ser aplicado em seqüência e tempo máximo pré-estipulados, a serem respeitados (Primi \& Almeida, 2000a, 2000b).

As provas foram corrigidas com o auxílio de crivos, somando-se os resultados e transformandoos em escores padrões e percentis com o auxílio de tabelas de conversão, que fornecem informações sobre as capacidades específicas dos participantes que somadas resultam em um escore total que fornece um parâmetro geral da sua capacidade de raciocínio.

O 16PF (Questionário dos Dezesseis Fatores de Personalidade) é um instrumento que fornece de forma objetiva uma completa cobertura das áreas da personalidade. As escalas que o compõem são independentes e fornecem um tipo de informação sobre o participante (Cattell, R.B., Cattell, A.K.S., \& Cattell, H.E.P. 1993; Russel \& Karol, 1999). Ele se apresenta na forma de questionário e é composto de 185 itens ordenados em 16 escalas (Primárias), tendo como objetivo aferir de forma geral a personalidade, sendo cada uma formada por 10 a 15 itens. Os dezesseis fatores primários medidos pelo teste, de acordo com Russel e Karol (1999) são: expansividade, inteligência, estabilidade emocional, afirmação, preocupação, consciência, desenvoltura, brandura, confiança, imaginação, requinte, apreensão, abertura a mudanças, auto-suficiência, auto-controle e tensão. Agrupamentos entre esses fatores formam os cinco Fatores globais: Extroversão, Ansiedade, 


\section{Cláudia Cobêro}

Rigidez de pensamento, Independência e Autocontrole.

O teste apresenta também escalas que constatam o estilo de respostas dadas pelo participante, podendo ser utilizado para complementar os resultados obtidos; de acordo com Russel e Karol (1999), eles são: Estilo AI-Administração da Imagem, composta por 12 itens que verificam a desejabilidade social, a propensão a agir de acordo com o que é esperado socialmente. Um alto índice neste item pode invalidar o teste, uma vez que indica que o participante não teria respondido de acordo com sua real personalidade. NF-Não - Freqüência, composta por 32 itens, que indicam se o tipo de resposta dada é parecido com o da maioria das pessoas; Estilo AQAquiescência, de 103 itens, que visa medir se o participante tende a responder sempre o verdadeiro ou o mais adequado.

O Kelly, Select Manual do 16PF (1999), Instrumento de Avaliação de Desempenho, contido no Apêndice $C$, tem como objetivo a coleta da opinião do participante e das pessoas com quem ele trabalha diariamente sobre suas ações profissionais, investigando aspectos como: conhecimento técnico, atitudes diante de tomada de decisões, reconhecimento pelo grupo, administração de conflitos, organização na realização das tarefas profissionais, abertura para mudanças, maneira de se comunicar e negociar, entre outros, sendo uma avaliação dividida em 2 fases: a primeira composta por quatro questões sobre o relacionamento no trabalho entre a pessoa que está respondendo e a que está sendo avaliada; a segunda por dez questões sobre o desempenho do avaliado comparado com o dos demais funcionários do seu setor, sendo pontuado da seguinte maneira: NDA = Nenhuma das Alternativas (uso da alternativa só se não houver informação para fornecer uma avaliação exata); 6 = excelente (melhor do que $90 \%$ dos trabalhadores com similar experiência); $5=$ muito bom (melhor do que $70 \%$ dos trabalhadores com similar experiência); 4 = bom (superior a metade dos trabalhadores com similar experiência); $\underline{3=}$ satisfatório (inferior à metade, mas aceitável comparado ao trabalhador com similar experiência); $\underline{2}=$ requer melhoria (um trabalhador que você não contrataria) e 1 = inaceitável (mau trabalhador).
Para o resultado ser mais confiável, este instrumento foi respondido por duas pessoas que, de alguma forma, apresentavam algum tipo de relação profissional com o mesmo (colegas de trabalho, supervisores e/ou subordinados). A pontuação final ficou constituída pela média dos pontos atribuídos pelos avaliadores em cada item do instrumento.

\section{Procedimentos}

A aplicação dos instrumentos na amostra foi realizada coletivamente, dentro da empresa em que trabalha e em uma sala cedida pela mesma. Em um primeiro momento os objetivos do estudo foram expostos e os termos de consentimento distribuídos. A amostra foi composta então pelos que retornaram o termo consentimento, com respostas afirmativas. Os instrumentos foram aplicados em dias diferentes, utilizando-se duas sessões: na primeira foi aplicado o $16 \mathrm{PF}$ e a primeira parte da BPR-5 e na segunda o instrumento MSCEIT, a Segunda parte da BPR-5 e o instrumento de avaliação de Desempenho Profissional.

\section{Resultados e Discussão}

Trabalhou-se primeiro verificando as correlações entre o teste MSCEIT e a BPR-5, MSCEIT, Avaliação de Desempenho e análise de regressão para verificar a validade incremental da inteligência emocional em prever o desempenho profissional. Na Tabela 1 são apresentadas as correlações de Pearson entre os oito subtestes da inteligência emocional com os coeficientes obtidos nas provas da BPR-5.

Tabela 1.Coeficientes de correlação de Pearson entre os subtestes do MSCEIT e da BPR-5

\begin{tabular}{|l|l|l|l|l|l|l|}
\hline & $\begin{array}{l}\text { RA- } \\
\text { raciocínio } \\
\text { abstrato }\end{array}$ & $\begin{array}{l}\text { RV- } \\
\text { raciocínio } \\
\text { verbal }\end{array}$ & $\begin{array}{l}\text { RM- } \\
\text { raciocínio } \\
\text { mecânico }\end{array}$ & $\begin{array}{l}\text { RE- } \\
\text { raciocínio } \\
\text { espacial }\end{array}$ & $\begin{array}{l}\text { RN- } \\
\text { raciocínio } \\
\text { numérico }\end{array}$ & $\begin{array}{l}\text { EG- } \\
\text { escore } \\
\text { geral }\end{array}$ \\
\hline FACES & $0,25^{* *}$ & 0,15 & 0,08 & $0,34^{* *}$ & 0,15 & $0,26^{* *}$ \\
\hline PAISAG & $0,24^{* *}$ & $0,19^{*}$ & 0,04 & 0,18 & $0,21^{*}$ & $0,22^{*}$ \\
\hline FACILIT & $-0,009$ & 0,01 & $-0,17$ & $-0,1$ & $-0,006$ & $-0,05$ \\
\hline SENSA & $0,25^{* *}$ & $0,27^{* *}$ & 0,17 & $0,23^{*}$ & $0,19^{*}$ & $0,30^{* *}$ \\
\hline TRANSI & 0,13 & $0,25^{* *}$ & 0,12 & $0,18^{*}$ & $0,24^{* *}$ & $0,22^{*}$ \\
\hline MISTUR & $0,33^{* *}$ & $0,31^{* *}$ & 0,17 & $0,40^{* *}$ & $0,18^{*}$ & $0,35^{* *}$ \\
\hline GERENC & 0,15 & $0,27^{* *}$ & 0,06 & $0,23^{*}$ & 0,08 & $0,20^{*}$ \\
\hline RELAC & $0,24^{*}$ & $0,27^{* *}$ & 0,15 & $0,19^{*}$ & 0,15 & $0,26^{* *}$ \\
\hline
\end{tabular}

Nota: $* \mathrm{p}<0,05 ; * * \mathrm{p}<0,01$

Observa-se que no geral há uma correlação de mínima para moderada entre o MSCEIT e a BPR5. Nota-se que a prova RM não se correlacionou, 
sendo esta uma habilidade específica, esperando-se assim que não houvesse correlação com os subtestes do MSCEIT. É possível observar também que o subteste facilitação não se correlacionou significativamente com os da BPR-5. Os coeficientes encontrados são coerentes com as reivindicações dos autores de que a inteligência emocional seja um tipo específico de inteligência já que as magnitudes situamse na faixa considerada moderada $(0,25-0,50)$, indicando uma convergência entre as medidas $(0,75$ $1,00)$ e nem muito baixas $(0-0,24)$ mostrando a independência dos construtos (Mayer \& Salovey, 1997, Mayer, Caruso \& Salovey, 2000, Mayer, Salovey \& Caruso, 2002a).

Na Tabela 2 são apresentadas os coeficientes de correlação entre os subtestes do MSCEIT com a avaliação de desempenho realizada pelo supervisor, pelo amigo e a geral obtida calculando-se a média das duas anteriores.

Tabela 2.Coeficientes de correlação de Pearson entre os escores do MSCEIT e a avaliação de desempenho

\begin{tabular}{|l|l|l|l|}
\hline & $\begin{array}{c}\text { Avaliação } \\
\text { supervisor }\end{array}$ & $\begin{array}{c}\text { Avaliação } \\
\text { colega }\end{array}$ & $\begin{array}{c}\text { Avaliação } \\
\text { geral }\end{array}$ \\
\hline FACES & 0,09 & $0,21^{*}$ & 0,17 \\
\hline PAISAG & 0,05 & 0,02 & 0,04 \\
\hline FACILIT & 0,03 & 0,08 & 0,06 \\
\hline SENSA & $0,23^{*}$ & 0,01 & 0,15 \\
\hline TRANSI & $0,20^{*}$ & 0,07 & 0,17 \\
\hline MISTUR & $0,27^{*}$ & 0,04 & $0,20^{*}$ \\
\hline GERENC & $0,34^{* *}$ & 0,11 & $0,28^{*}$ \\
\hline RELAC & $0,22^{*}$ & 0,07 & 0,18 \\
\hline
\end{tabular}

Nota: $* \mathrm{p}<0,05 ; * * \mathrm{p}<0,01$

Observa-se a existência de coeficientes de correlação significativos e positivos indicando que as pessoas com maior Inteligência Emocional tendem a receber avaliações mais positivas das que convivem com elas; e na primeira avaliação de desempenho (supervisor) as correlações são em maior número e superiores se comparadas com a segunda (colega). $\mathrm{Na}$ avaliação do supervisor os subtestes gerenciamento e misturas foram os mais significativos. Podese assim concluir que a inteligência emocional contribui para a predição do desempenho profissional, mas com ressalvas, pois só se obteve correlação do desempenho geral em dois dos oito subtestes que compõem a inteligência emocional.

Sabendo-se que a inteligência emocional associa-se ao desempenho profissional e que ela também está correlacionada com a inteligência, podese questionar se a previsão do desempenho é a compartilhada com a inteligência. Caso isso seja verdadeiro, a emocional não apresentaria informações únicas para a previsão do desempenho profissional, ou seja, não teria validade incremental (Smith, Fischer \& Fister, 2003). Consequentemente, não poderia ser considerada uma habilidade distinta da geral a prever o desempenho profissional.

Para testar essa hipótese foi feita uma análise de regressão em dois blocos tendo como variável dependente, a ser prevista, o desempenho profissional avaliado pelo supervisor já que essa medida apresentou-se com coeficientes de dispersão maiores e menor efeito teto $(\mathrm{M}=4,38, \mathrm{DP}=0,96)$ se comparada à avaliação dos colegas e a medida composta pela média das duas $(M=4,58$ e $M=4,48 ; D P=0,85$ e $\mathrm{DP}=0,75$, respectivamente). No primeiro bloco da análise, entrou-se com o escore global da BPR-5 como variável independente representando a inteligência geral. No segundo bloco com duas variáveis independentes do MSCEIT, os escores fatoriais da área experiencial (IEE) e estratégica (IES, ver Tabela 1 para informações sobre a composição desses escores) já que estudos prévios indicam que um modelo de dois fatores é a melhor estrutura para representar os subtestes do MSCEIT na amostra brasileira (Cobêro, 2004; Dantas \& Noronha, 2005), utilizando-se o método de entrada na equação passo a passo (stepwise o $\mathrm{p}<0,05$ para entrada) buscandose verificar quais variáveis do MSCEIT: IEE e/ou IES contribuiriam significativamente para a previsão do desempenho.

Nesse segundo bloco da análise testa-se a capacidade preditiva "única" da IE para além do que eventualmente pudesse ter sido previsto pela BPR5 , já que a variância restante, para a análise no segundo bloco, consiste em um resíduo obtido depois de eliminada a covariância BPR-5 X desempenho. A eventual associação significativa no segundo bloco representa covariância única entre IE e desempenho. 


\section{Cláudia Cobêro}

Na Tabela 3 são apresentados os resultados globais dos dois blocos e na Tabela 4 os coeficientes da equação de regressão e outras estatísticas concernentes a essa análise.

Tabela 3.Resultados gerais da análise de regressão buscando prever o desempenho a partir da BPR-5 e do MSCEIT

\begin{tabular}{|l|r|r|r|r|r|r|r|}
\hline Modelo & \multicolumn{1}{|c|}{ R } & \multicolumn{1}{|c|}{$\mathbf{R}^{2}$} & Mud. R $^{2}$ & F & G I 1 & G I 2 & Sig \\
\hline Bloco 1. BPR-5 & 0,32 & 0,099 & 0,091 & 12,9 & 1 & 118 & 0 \\
\hline Bloco 2 BPR-5 + IES & 0,4 & 0,16 & 0,146 & 11,2 & 2 & 117 & 0 \\
\hline
\end{tabular}

Tabela 4. Coeficientes da equação de regressão buscando prever o desempenho a partir da BPR-5 e do MSCEIT

\begin{tabular}{|c|c|c|c|c|c|}
\hline \multirow{2}{*}{ Bloco } & \multicolumn{2}{|c|}{$\begin{array}{c}\text { Coef. } \\
\text { não padron. }\end{array}$} & \multirow{2}{*}{\begin{tabular}{|c|} 
Coef. \\
Padron \\
Beta \\
\end{tabular}} & & \multirow[t]{2}{*}{ Sig. } \\
\hline & B & erro & & & \\
\hline constante & 3,321 & 0,31 & & 10,7 & \\
\hline BPR-5 & 0,018 & 0,005 & 0,315 & 3,6 & \\
\hline constante & 1,845 & 0,588 & & 3,14 & 0,002 \\
\hline BPR-5 & 0,012 & 0,005 & 0,215 & 2,36 & 0,02 \\
\hline IES & 0,045 & 0,015 & 0,266 & 2,92 & 0,004 \\
\hline
\end{tabular}

Como pode ser observado o resultado da regressão foi significativo $(\mathrm{F}[2,117]=11,16$; $\mathrm{p}<$ $0,001)$. No primeiro bloco o escore global da BPR-5 apresenta uma associação significativa com o desempenho $(\mathrm{r}=0,31)$ explicando aproximadamente $10 \%$ da variância da avaliação de desempenho. No segundo bloco das duas variáveis inseridas na análise o escore IES (área estratégica composta pela soma dos subtestes Transições, Misturas, Administração de Emoções e Relações Emocionais) foi incorporado na equação aumentando o R para 0,40, explicando cerca de $6 \%$ adicionais da variância do desempenho, sendo este incremento significativo (ver Tabela 3). A mesma análise foi feita incluindo os fatores do 16PF no primeiro bloco; nenhum fator de personalidade estava associado significativamente ao desempenho. Somente o Fator Inteligência (Fator B) associou-se ao desempenho, o que já era esperado, pois assim como a BPR-5 esse fator consiste em uma prova de inteligência. Nesta análise os resultados foram semelhantes aos encontrados com a BPR-5 sendo que a área estratégica explicou um montante adicional e significativo da variância após o fator B ter entrado na equação. Esses resultados demonstram que a área estratégica do MSCEIT possui validade incremental na previsão do desempenho dos participantes além do que advindo das medidas tradicionais de inteligência.

Portanto, revendo as questões desse trabalho, isto é, se a inteligência emocional se correlacionaria com desempenho profissional, e se isto poderia ser explicado por um fator específico diferente dos cognitivos já conhecidos, é possível dizer que sim, ela está associada ao desempenho profissional de maneira única. Um primeiro ponto a ser mencionado é que os fatores medidos no MSCEIT aparecem como medidas cognitivas distintas do BPR-5 ainda que associados à inteligência. Além disso, após remover, da variância da medida de desempenho, a parcela tradicionalmente associada à inteligência, a medida IES contribuiu significativamente para a previsão do desempenho, e esta é única (incremental) já que no segundo bloco da análise de regressão a variância da medida de desempenho já está expurgada do componente ligado à inteligência e a parcela comum entre esta e a emocional. Portanto qualquer parcela significativa explicada no segundo bloco significa variância única às medidas de IE.

Por outro lado a magnitude da associação entre inteligência emocional e desempenho não chega a ser significativamente maior do que a observada entre a geral e desempenho. Assim esse estudo corrobora os dados da literatura internacional mostrando que a inteligência geral está associada significativamente ao desempenho no trabalho (Barrett \& Depinet, 1991; Kuncel, Hezlett \& Ones, 2004; Wilhelm \& Engle, 2005). Também demonstra a validade de critério da BPR-5 no contexto organizacional, atestando sua capacidade em prever desempenho. Ao mesmo tempo mostra que a inteligência emocional é um preditor único do desempenho no trabalho, mas vista como fator adicional e não o mais importante, como diz Goleman (1995). Em suma, os dados desse trabalho trazem mais evidências positivas coerentes com as reivindicações de que inteligência emocional é um tipo separado das inteligências conhecidas e importantes no contexto organizacional. Também atesta a validade da BPR-5 para prever o desempenho e mostra que usados conjuntamente esses instrumentos aumentam a capacidade preditiva dele. 


\section{Análise correlacional do MSCEIT com o 16 PF}

A Tabela 5 traz as correlações encontradas entre os 16 fatores do $16 \mathrm{PF}$ e os oito subtestes do MSCEIT.

Observam-se correlações significativas existentes entre o Fator B (inteligência) com quatro dos subtestes do MSCEIT, o que mais uma vez reforça a concepção da inteligência emocional como um tipo de inteligência. A maior correlação de B foi com o subteste misturas. O Fator M (imaginação) apresenta correlação negativa com a maioria dos subtestes da inteligência emocional, quais sejam, Paisagem, Facilitação, Transição, Gerenciamento das emoções e Relações emocionais; com os demais subtestes ele não demonstrou correlação. Esses dados sugerem que quanto mais imaginativa uma pessoa for, menos inteligente emocionalmente ela tende a ser. Essa correlação com o Fator $M$ também foi encontrada por Bueno e Primi (2003) que trabalharam com o MEIS

Tabela 5.Coeficientes de correlação de pearson entre os escores dos subtestes do MSCEIT com os fatores do 16PF e pode indicar que pessoas cuja atenção esteja mais voltada para o ambiente e suas sensações ao invés do contato com o mundo interno das idéias e sentimentos tenham maior inteligência emocional, o que se associaria à forma como o MSCEIT operacionaliza a avaliação empregando o consenso como grade de correção, que talvez indique que o MSCEIT esteja medindo uma capacidade interpessoal de entendimento das convenções sociais sobre o comportamento das pessoas, mais do que a visão intrapessoal coerente com um processamento intuitivo associado a valores altos no Fator M.

No geral, pode-se dizer que ocorreram poucas correlações significativas e essas foram também de magnitudes baixas, sendo a maioria menor de 0,25 , observando-se que não houve sobreposição entre o MSCEIT com traços de personalidade. Esses dados corroboram estudos brasileiros e estrangeiros que sustentam a posição de que, quando medida por testes de desempenho, a inteligência emocional e personalidade são construtos distintos (Bueno, 2002, Bueno \& Primi, 2003; Brackett \& Mayer, 2003;

\begin{tabular}{|l|l|l|l|l|l|l|l|l|}
\hline & FACES & PAISAG & FACILIT & SENSA & TRANSI & MISTUR & GERENC & RELAC \\
\hline AI - adm. da imagem & $-0,02$ & $0,22^{*}$ & $0,23^{*}$ & 0,09 & 0,05 & 0,06 & 0,18 & 0,08 \\
\hline NF não - freqüência & $-0,16$ & 0,03 & 0,01 & $-0,15$ & 0,02 & $-0,1$ & $-0,09$ & $-0,08$ \\
\hline AQ aquiescência & 0,03 & $-0,17$ & $-0,07$ & 0,04 & $-0,12$ & 0,06 & $-0,09$ & $-0,01$ \\
\hline A expansividade - geral & $-0,02$ & $-0,04$ & $-0,09$ & 0,05 & 0,15 & $-0,01$ & $-0,03$ & $-0,15$ \\
\hline B inteligência & $0,25^{* *}$ & 0,04 & $-0,07$ & $0,23^{*}$ & $0,22^{*}$ & $0,34^{* *}$ & 0,17 & 0,17 \\
\hline C estabilidade emocional & 0,08 & $0,23^{*}$ & $0,19^{*}$ & 0,02 & 0,13 & 0,12 & $0,26^{* *}$ & 0,02 \\
\hline E afirmação - geral & $-0,05$ & $-0,12$ & 0,01 & $-0,11$ & $-0,08$ & 0,03 & $-0,18$ & $-0,18$ \\
\hline F preocupação & 0,08 & $-0,03$ & $-0,07$ & 0,14 & 0,07 & 0,05 & 0,11 & $-0,12$ \\
\hline G consciência & 0,13 & 0,17 & $-0,03$ & 0,15 & $-0,01$ & 0,11 & 0,12 & $0,25^{* *}$ \\
\hline H desenvoltura & 0,04 & 0,18 & $-0,03$ & 0,09 & 0,14 & 0,06 & $-0,05$ & 0,01 \\
\hline I brandura - geral & 0,06 & 0,03 & 0,14 & 0,12 & 0,08 & $0,18^{*}$ & 0,17 & $-0,05$ \\
\hline L confiança & 0,01 & $-0,14$ & $-0,17$ & 0,09 & $-0,08$ & 0,01 & $-0,19^{*}$ & $-0,06$ \\
\hline M imaginação & $-0,13$ & $-0,19^{*}$ & $-0,25^{* *}$ & $-0,17$ & $-0,20^{*}$ & $-0,16$ & $-0,19^{*}$ & $-0,25^{* *}$ \\
\hline N requinte & 0,1 & 0,05 & $-0,07$ & $-0,02$ & $-0,06$ & $-0,01$ & 0,02 & $0,29^{* *}$ \\
\hline O apreensão & 0,11 & 0,01 & $-0,1$ & 0,1 & 0,16 & 0,07 & $-0,04$ & 0,05 \\
\hline Q1 abertura a mudanças & $-0,02$ & $-0,1$ & $-0,07$ & 0,11 & 0,06 & 0,11 & $-0,03$ & 0,11 \\
\hline Q2 auto-suficiência & $-0,07$ & $-0,02$ & $-0,05$ & $-0,02$ & $-0,03$ & $-0,06$ & $-0,21^{*}$ & 0,05 \\
\hline Q3 auto-controle & 0,02 & 0,06 & 0,11 & 0,1 & $-0,01$ & 0,11 & 0,06 & 0,07 \\
\hline Q4 tensão & 0,07 & $-0,09$ & $-0,19^{*}$ & 0,06 & $-0,01$ & 0,05 & $-0,08$ & $-0,08$ \\
\hline fator I - extroversão & 0,02 & 0,01 & $-0,02$ & 0,09 & 0,13 & 0,05 & 0,08 & $-0,20^{*}$ \\
\hline fator II - ansiedade & 0,04 & $-0,16$ & $-0,23^{*}$ & 0,08 & $-0,02$ & 0,01 & $-0,20^{*}$ & $-0,03$ \\
\hline fator III - rigidez de pensamento & 0,02 & 0,12 & 0,06 & $-0,11$ & $-0,06$ & $-0,13$ & $-0,02$ & 0,08 \\
\hline fator IV - independência & $-0,03$ & $-0,09$ & $-0,08$ & 0,02 & $-0,01$ & 0,07 & $-0,19^{*}$ & $-0,09$ \\
\hline fator V - auto-controle & 0,1 & $0,19^{*}$ & 0,15 & 0,15 & 0,04 & 0,15 & 0,13 & $0,27^{* *}$ \\
\hline
\end{tabular}

Nota: * $\mathrm{p}<0,05 ; * * \mathrm{p}<0,01$ 
Bedwell, sd.; Dantas, 2004; Lopes, Brackett, Nezlek, Schutz, Sellin \& Salovey, 2004; Mayer, Salovey, Caruso \& Sitarenios, 2001,2003; Nascimento, 2006 e Primi, Bueno \& Muniz, 2006)

\section{Considerações finais}

Esse estudo cumpriu seu objetivo de verificar a validade de critério do MSCEIT com desempenho profissional, além de investigar sua validade convergente com medidas de inteligência e divergente em relação a medidas de traços de personalidade. Observando os resultados obtidos e os outros citados, pode-se concluir que a inteligência emocional, avaliada pelo MSCEIT, que é um teste seguindo o modelo de desempenho, é um construto novo associado, mas distinto da inteligência e praticamente distinto de medidas de personalidade.

Com relação à validade de critério do MSCEIT, conclui-se que ele pode ser um preditor do desempenho profissional trazendo informações únicas e complementares em relação ao que medidas tradicionais de inteligência provêm. Diz-se, então, que a probabilidade de uma pessoa inteligente emocionalmente ter melhor desempenho no trabalho é maior do que de uma com baixa IE. Ao mesmo tempo esse estudo traz dados que confirmam as relações relatadas na literatura entre inteligência e desempenho no trabalho, apresentando evidências de validade de critério para a BPR-5 e para o Fator B do 16PF para previsão de desempenho no trabalho, e relata validade de critério no contexto organizacional de uma bateria de medida de inteligência.

Assim sendo, em termos gerais, é preciso reforçar a idéia argumentada por Goleman (1998) que os parâmetros de avaliação do mercado de trabalho devem considerar aspectos sobre como as pessoas lidam consigo e com os outros além da inteligência e da formação acadêmica. Entretanto isso não quer dizer que a inteligência tradicionalmente definida e medida não seja importante. As duas variáveis, inteligência geral e emocional são igualmente importantes na previsão do desempenho. Assim a capacidade de resolver problemas complexos usando o raciocínio indutivo-dedutivo como medido na BPR-5 (Primi \& Almeida, 2000b) e a habilidade de entender as informações sobre o mundo trazidas pelas emoções e a habilidade de as gerenciar em si e nos outros são aspectos importantes do desempenho no trabalho, já que pessoas com essas características tendem a ser mais frequentemente avaliadas, pelos seus supervisores, como funcionários muito bons ou excelentes.

Conclui-se então, que o construto de inteligência emocional parece ser um tipo específico de inteligência, que antes não havia sido demonstrado, sendo independente de medidas de personalidade e relativamente associado a medidas tradicionais de inteligência podendo ainda ser útil na previsão do desempenho profissional.

\section{Referências}

Anastasi, A., \& Urbina, S. (2000). Testagem Psicológica. Porto Alegre: Artmed.

Barret, G., \& Depinet, R. L. (1991). A reconsideration of testing for competence ratherthan for intelligence. American Psychologist, 46 (10), 1012-1024.

Bedwell, S. (s.d). Emotional intelligence: personality revisited or something else? Symposium conducted at the Annual meeting of the Society of Industrial and Organizational Psychology, Orlando FL.Disponível em: 〈http://www.ipat.com/ pdf/ejisiop2003.pdf>.

Brackett, M. A., \& Mayer, J. D. (2003). Convergent, discriminant and incremental validity of competing measures of emotional intelligence. Personality and Social Psychology Bulletin, 29 (9), $1147-1158$.

Bueno, J. M. H. (2002). Inteligência emocional: um estudo de validade da capacidade de perceber emoções. Dissertação de Mestrado, Universidade São Francisco, Itatiba.

Bueno, J. M. H., \& Primi, R. (2003). Inteligência Emocional: um estudo de validade sobre a capacidade de perceber emoções. Psicologia Reflexão e Crítica,16 (2), 279-291.

Cattell, R. B., Cattell, A. K. S., \& Cattell, H. E. P. (1993). Questionário 16PF Quinta Edição. Rio de Janeiro: CEPA.

Cobêro, C. (2004). Inteligência emocional: validade do MSCEIT no contexto organizacional. Dissertação de Mestrado, Universidade São Francisco. Itatiba. 
Dantas, M .A. (2004). Evidências de validade do Mayer Salovey Caruso Emotional Intelligence Test (MSCEIT). Dissertação de Mestrado, Universidade São Francisco, Itatiba.

Dantas, M. A., \& Noronha, A. P. P. (2005). Inteligência Emocional: Parâmetros Psicométricos de Um Instrumento de Medida. Estudos e Pesquisas em Psicologia 5, (1), 59-72.

Goleman, D. (1995). Inteligência Emocional. Rio de Janeiro: Objetiva.

Goleman, D. (1998). Trabalhando com a Inteligência Emocional. (Trad. M. H. C. Cortês). Rio de Janeiro: Objetiva.

Hein, S. (2003). Definition and history of emotional intelligence. Disponível em: <http:// www.eqi.org/history.htm>. Acesso em 19 de Abril de 2003.

Jesus Junior, A. G. (2004). Estudo de validade e precisão do Mayer - Salovey - Caruso Emotional Intelligence Test. Dissertação de Mestrado, Universidade São Francisco, Itatiba.

Kelly, M. L. (1999). 16 PF Select Manual. EUA: Illinois - Institute for Personality and Ability Testing (IPAT).

Kuncel, N. R., Hezlett, S. A., \& Ones, D. S. (2004). Academic performance, career potential, creativity and job performance: can one construct predict them all? Journal of Personality and Social Psychology, 86, 148-161.

Lopes, P. N., Brackett, M. A., Nezlek, J., Schutz, I., Sellin, I., \& Salovey, P. (2004). Emotional intelligence and social interaction. Personality and Social Psychology Bulletin, 30(8), 1018-1034.

Mayer, J. D., Caruso, D. R., \& Salovey, P. (2000). Emotional intelligence meets traditional standards for an intelligence. Intelligence, 27 (4), 267-298.

Mayer, J. D., DiPaolo, M.T., \& Salovey, P. (1990). Perceiving affective content in ambiguous visual stimuli: A component of emotional intelligence. Journal of Personality Assessment, 54, 772-781.

Mayer, J. D., \& Salovey, P. (1993). The intelligence of emotional intelligence. Intelligence, 17(4), 433-442.
Mayer, J. D., \& Salovey, P. (1997). What is emotional intelligence? In P. Salovey \& D. J. Sluyter (Eds.), Emotional Development and Emotional Intelligence: Implications for Educators (pp. 3-31). New York: Basic Books.

Mayer, J. D.; Salovey, P., \& Caruso, D. (2002a). Mayer-Savoley-Caruso Emotional Intelligence Test: Users Manual. Toronto, CA: Multi-Health Systems.

Mayer, J. D.; Salovey, P., \& Caruso, D. (2002b). Mayer-Savoley-Caruso Emotional Intelligence Test. Toronto, CA: Multi-Health Systems.

Mayer, J. D.; Salovey, P.; Caruso, R. D., \& Sitarenios, G. (2001). Emotional intelligence as a standard intelligence. Emotion, 1 (3), 232-242.

Mayer, J. D.; Salovey, P.; Caruso, D., \& Sitarenios, G. (2003). Measuring emotional intelligence with the MSCEIT V 2.0. Emotion, 3 (1), 97-105.

Nascimento, E. (2000). Adaptação e Validação do Teste WAIS-III para Contexto Brasileiro. Dissertação de Doutorado, Universidade de Brasília, Brasília.

Nascimento, M. M. (2006). Evidências de Validade Para o Teste de Inteligência Emocional MSCEIT em Policiais. Dissertação de Mestrado, Universidade São Francisco, Itatiba.

Primi, R., \& Almeida, L.S. (1998). Baterias de Provas de Raciocínio-BPR-5. São Paulo: Casa do Psicólogo.

Primi, R., \& Almeida, L. S. (2000a). Estudo de Validação da Bateria de Provas de Raciocínio (BPR-5). Psicologia: Teoria e Pesquisa, 16 (2), 165-173.

Primi, R., \& Almeida, L. S. (2000b). Baterias de Provas de Raciocínio (BPR-5): Manual técnico. São Paulo: Casa do Psicólogo.

Primi, R., Bueno, J. M. H., \& Muniz, M. (2006). Inteligência emocional: validade convergente e discriminante do MSCEIT com a BPR-5 e o 16PF. Revista Psicologia Ciência e Profissão, 26 (1), 26-45. 
Roberts, R. D.; Flores-Mendoza, C. E., \& Nascimento, E. (2002). Inteligência Emocional: Um Construto Científico? Cadernos de Psicologia e Educação Paidéia, 12 (23), 77-92.

Russel, M., \& Karol, D. (1999). 16PF Quinta Edição Manual do Examinador. Rio de Janeiro: CEPA.

Salovey, P., \& Mayer, J. D. (1990). Emotional intelligence. Imagination, Cognition and Personality, 9, 185-221.

Smith, G. T., Fischer, S., \& Fister, S. M. (2003). Incremental Validity Principles in Test Construction . Psychological Assessment, 15, 467-477.

Wilhelm, O., \& Engle, R. W. (2005). Intelligence: a diva and a workhorse. In O. Eilhelm \& R.W. Engle (Eds.), Handbook of Understanding and Measuring Intelligence. (pp.1-9). Thousand Oaks, CA: SAGE.

As atividades de pesquisa que deram origem a esse artigo foram financiadas pelo $\mathrm{CNPq}$ (primeiro e segundo autor). 